\title{
Archéologie du paysage de la plaine de Caen du Néolithique à l'époque mérovingienne
}

Laurent Lespez, Cécile Germain-Vallée et Xavier Savary

\section{QpenEdition \\ Journals}

Édition électronique

URL : http://journals.openedition.org/adlfi/7488

ISSN : 2114-0502

Éditeur

Ministère de la culture

Référence électronique

Laurent Lespez, Cécile Germain-Vallée et Xavier Savary, « Archéologie du paysage de la plaine de Caen du Néolithique à l'époque mérovingienne ", ADLFI. Archéologie de la France - Informations [En ligne],

Basse-Normandie, mis en ligne le 01 mars 2007, consulté le 02 mai 2019. URL : http:// journals.openedition.org/adlfi/7488

Ce document a été généré automatiquement le 2 mai 2019.

(c) Ministère de la Culture et de la Communication, CNRS 


\title{
Archéologie du paysage de la plaine de Caen du Néolithique à l'époque mérovingienne
}

\author{
Laurent Lespez, Cécile Germain-Vallée et Xavier Savary
}

Date de l'opération : 2007 (PT)

Inventeur(s) : Lesprez Laurent (SUP) ; Germain-Vallée Cécile ; Savary Xavier

1 Le projet collectif de recherche intitulé « Archéologie du paysage de la plaine de Caen du Néolithique à l'époque mérovingienne " s'inscrit dans l'ensemble des recherches sur les dynamiques des paysages sur la longue durée qui se sont multipliées en France au cours de ces dernières années.

2 Ce projet de recherche étudie la plaine de Caen qui constitue la partie la plus occidentale du bassin de Paris. La zone d'étude choisie occupe une surface d'environ $1240 \mathrm{~km}^{2}$ et délimite un espace d'une cinquantaine de kilomètres de longueur du nord au sud pour une largeur ouest-est de presque trente kilomètres (Fig. $\mathrm{n}^{\circ} 1$ : Archéologie du paysage de la plaine de Caen du Néolithique à l'époque mérovingienne). Cet espace s'impose pour le type d'étude envisagé par son potentiel archéologique et paléoenvironnemental. En effet, un nombre très important de découvertes archéologiques a été effectué ces dernières années à l'occasion de nombreuses fouilles préventives et programmées résultant, en particulier, de la croissance de l'agglomération caennaise et des travaux routiers pour la desservir.

Ces fouilles auxquelles se sont ajoutées régulièrement, depuis vingt ans maintenant, des prospections aériennes systématiques, fournissent de nombreuses données sur les habitats et la mise en valeur de l'espace du Néolithique à nos jours et permettent d'envisager une étude des facteurs environnementaux de l'habitat et des conséquences des modes de peuplement et de mise en valeur sur les paysages. 
4 Enfin, l'importance des archives sédimentaires dans les vallées qui l'encadrent (Dives, Seulles) ou la traversent (Orne, Seulles, Laizon) ont permis de produire de nouvelles et nombreuses données paléoenvironnementales sur la dynamique des paysages depuis le Néolithique.

5 C'est dans cette perspective, alliant un espace riche d'informations archéologiques et paléoenvironnementales et une approche pluridisciplinaire sur la longue durée encore rarement pratiquée dans les espaces de plaines et plateaux dans la partie occidentale du bassin de Paris, que se positionne ce programme collectif de recherche. La période étudiée court du Néolithique jusqu'à la fin de l'époque mérovingienne (fin du $\mathrm{VII}^{\mathrm{e}} \mathrm{s}$.). Elle doit permettre d'étudier le rôle fondamental de la néolithisation puis des transformations des systèmes socio-économiques et plus particulièrement des périodes de croissance démographique et de mise en valeur agricole ou à l'inverse de déprise ou de réorganisation des systèmes agraires dans les dynamiques paysagères au cours de la Protohistoire et des premières époques historiques.

6 La recherche s'appuie sur une méthodologie en trois temps : inventaire, confrontation, nouvelles investigations. En 2007, la phase d'inventaire a été entamée avec un recensement des études géoarchéologiques intra-sites prenant en compte les différentes études spécialisées informant sur les paysages anciens.

7 Parallèlement, a été conduit l'inventaire des données disponibles hors-sites, acquises par des investigations paléoenvironnementales pratiquées dans les archives naturelles que constituent les fonds de vallée. Cela concerne plus précisément les données géomorphologiques et pédologiques, archéobotaniques (palynologiques, anthracologiques, carpologiques et xylologiques) et archéozoologiques et les analyses portant sur les matières premières en terre, publiées et non publiées, exploitées ou non par les rapports de fouilles. Au total, soixante et onze entités archéologiques ayant fait l'objet d'une ou plusieurs études paléoenvironnementales et dix-huit entités paléoenvironnementales ont été recensées. Il s'agissait de la première étape d'un travail consistant à créer une base de données géoarchéologiques et paléoenvironnementales accessible à tous.

8 Parallèlement, une première réflexion collective a permis de construire l'architecture de la base de données. Elle a associé archéologues et spécialistes du service régional d'Archéologie de Basse Normandie, du service départemental d'archéologie du Calvados, de l'INRAP, du CRAVO et des universités de Caen (GEOPHEN-UMR 6554 CNRS ; CRAHMUMR 6577 CNRS) et de Rennes 1 (CREAAH-UMR 6566 CNRS). La base de données proposée joint deux bases de données correspondant aux deux types d'entités recensées et est articulée autour de la base de données et du système géographique PATRIARCHE de la Carte archéologique (SRA Basse-Normandie).

9 Par ailleurs, le dialogue interdisciplinaire autour des relations entre les Sociétés et la Nature à travers la gestion des ressources environnementales et de ses conséquences paysagères, a été entamé à l'occasion de deux séminaires organisés à la MRSH de Caen le 11 juin et le 24 septembre. Ils ont permis de réunir des archéologues, géoarchéologues et paléoenvironnementalistes, de préciser les enjeux fondamentaux de la recherche et d'identifier une communauté de chercheurs intéressés par la problématique et de constituer, au-delà de l'équipe de coordination, une équipe d'animation rassemblant des chercheurs d'horizons différents, prêts à s'investir durablement dans le programme. 

conduite dans la vallée de la Seulles. Elle permet de compléter les données acquises jusqu'à présent dans les vallées qui encadrent ou traversent la plaine de Caen et souligne, en particulier, le rôle du premier millénaire avant notre ère dans les colmatages limoneux qui caractérisent tous les fonds de vallées étudiés.

11 LESPEZ Laurent, GERMAIN-VALLÉE Cécile et SAVARY Xavier

\section{ANNEXES}

Fig. $\mathrm{n}^{\circ} 1$ : Archéologie du paysage de la plaine de Caen du Néolithique à l'époque mérovingienne

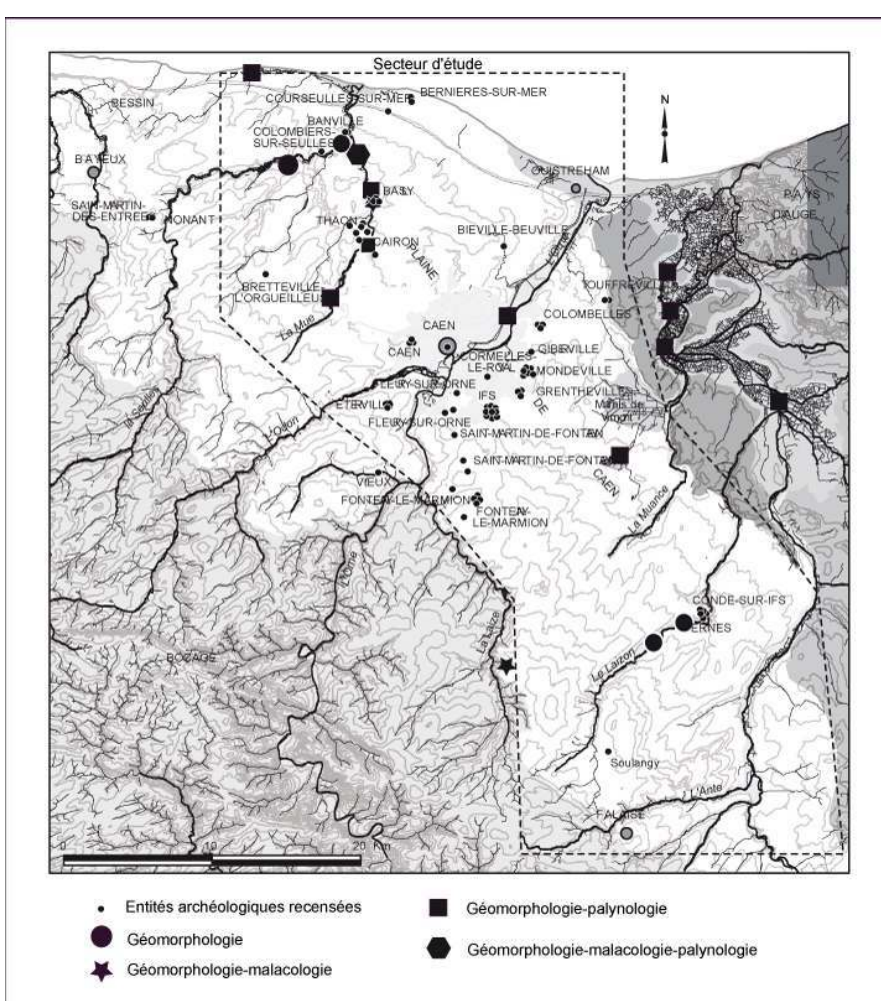

Auteur(s) : Lesprez, Laurent. Crédits : Lesprez, Laurent (2007)

\section{INDEX}

Index chronologique : Antiquité romaine, ép. méroïtique, ép. mérovingienne, Néolithique, Protohistoire

operation Prospection thématique (PRT)

Index géographique : Basse-Normandie, Calvados (14), Caen 
AUTEURS

LAURENT LESPEZ

SUP 